\title{
Controle Preditivo Robusto com Horizonte Finito de sistemas lineares variantes no tempo
}

\author{
Ary M. Batista*, Humberto X. de Araújo* \\ * Programa de Pós Graduação em Engenharia Elétrica, Universidade Federal da Bahia - UFBA,Rua Aristides Novis, 02, \\ CEP 40210-630 - Salvador, BA, Brasil.(arymontenegrol@hotmail.com, humberto.araujo@ufba.br)
}

\begin{abstract}
This work proposes a new approach for the Finite Horizon Robust Model Predictive Control (FH-RMPC) problem, for linear discrete systems with time-varying polytopic uncertainties. The basic idea of this approach is to find, in each sampling time, the optimal state feedback control law based on Riccati difference equations (RDE) that minimizes an objective function with finite horizon and comply with input and output constraints. The closed loop stability, known as one of the challenging themes of FH-RMPC strategy with constraints, is based on monotonic property of the RDE from Linear Quadratic (LQ) control problem with finite horizon. In this proposed method it is not necessary that the usual terminal weighting matrix be fixed to achieve asymptotic stability. Finally, the efficiency of the proposed approach is illustrated by numerical examples.
\end{abstract}

Resumo: Este trabalho propõe uma nova abordagem para o problema de Controle Preditivo Robusto baseado em modelo de Horizonte Finito (FH-RMPC), de sistemas lineares em tempo discreto com incertezas politópicas variantes no tempo. A ideia básica desta abordagem é encontrar, em cada instante de amostragem, um controlador ótimo por realimentação de estado, basedo nas equações a diferenças de Riccati (RDE), que minimiza uma função custo de horizonte finito e atenda as restrições nos sinais de controle e saída. A garantia de estabilidade em malha fechada, conhecida como um dos temas mais desafiadores da estratégia FH-RMPC com restrições, é baseada na propriedade de monotonicidade das RDEs obtidas na solução do problema de Controle Linear Quadrático (LQ) de horizonte finito. Na abordagem proposta não é necessário que a usual matriz de custo terminal seja fixa para atingir a estabilidade assintótica. Finalmente, a eficiência do método proposto é ilustrada por exemplos numéricos.

Keywords: Robust Predictive Control, Stabilty guarantee, Time-varying Uncertainties, State Feedback, Riccati Difference Equation, Linear Matrix Inequalities.

Palavras-chaves: Controle Preditivo Robusto, Garantia de estabilidade, Incertezas variantes no tempo, Realimentação de estado, Equações a diferença de Riccati, Desigualdades Matriciais Lineares.

\section{INTRODUÇÃO}

O Controle Preditivo Baseado em Modelo (MPC - Model Predictive Control), também referenciado como controle de horizonte deslizante (Moving Horizon Optimal Control) engloba uma classe de controladores on-line que computam iterativamente o controle ótimo baseado em um modelo da planta. Uma limitação do MPC é não levar em consideração explicitamente as incertezas inerentes ao modelo da planta, o que torna o desempenho e a estabilidade muito dependentes da precisão do modelo. Um trabalho relevante que supera tal limitação foi desenvolvido por Khotare et al. (1996). Neste, uma técnica de Controle Preditivo Robusto de Horizonte Infinito (IH-RMPC - Infinity Horizon Robust Model Predictive Control), que incorpora as incertezas na modelagem da planta e garante estabilidade quadrática em malha fechada, utilizando desigualdades matriciais lineares (LMI), foi apresentado. Desde então, diversas trabalhos, como o de Cuzzola et al. (2002), Mao (2003), Casavola et al.
(2004) e Wada e Saeki (2006), surgiram de forma a aprimorar tal técnica IH-RMPC.

Cuzzola et al.(2002) apresentaram uma melhoria na técnica desonvolvida em Khotare et al. (1996), propondo uma abordagem menos conservadora a partir dos resultados de Oliveira et al. (1999), para obter a estabilidade com funções de Lyapunov dependentes de parâmetros. Mao (2003) reavaliou os resultados de Cuzola et al. (2002), estendendoos para os sistemas variantes no tempo (LTV).

Ao contrário da estratégia IH-RMPC que foi bem investigada, a estratégia de Controle Preditivo Robusto de Horizonte Finito (FH-RMPC - Finite Horizon Robust Model Predictive Control) ainda necessita de mais estudos para contornar alguns obstáculos de forma satisfatória, como por exemplo: a complexidade computacional das predições de estados e saídas futuras em função das incertezas e a garantia de estabilidade. 
Em Kim (2002), foi proposto um algoritmo FH-RMPC para sistemas discretos variantes no tempo utilizando LMI. O esquema de controle é baseado em um problema de otimização da função custo com horizonte finito e matriz de custo terminal para garantir estabilidade em malha fechada.

Em Chu et al. (2006), um algoritmo FH-RMPC robusto em relação às incertezas do modelo foi apresentado. É utilizada uma abordagem LMI com uma matriz de média móvel do sistema para representar o modelo das incertezas, o qual difere das incertezas estruturadas convencionais. A estabilidade é obtida através de matriz custo terminal.

Kanev et al. (2006) propõem um algoritmo FH-RMPC com garantia de estabilidade assintótica em malha fechada pela imposição de restrição no sinal de controle no final do horizonte de predição.

De forma geral, a estabilidade das técnicas FH-RMPC presentes na literatura é garantida através de restrições terminais como pode ser observado em Chu et al. (2006) e Kim (2002), ou através de restrição no sinal de controle ao final do horizonte de predição, como em Kanev et al. (2006).

Este trabalho propõe uma estratégia FH-RMPC para sistemas lineares em tempo discreto com incertezas politópicas variantes no tempo. Nesta abordagem, são utilizadas restrições LMI obtidas através das equações recursivas de Riccati para encontrar, a cada instante $k$, uma lei de controle por realimentação de estado. Não são utilizadas restrições terminais nesta estratégia.

Na Seção 2, a formulação do FH-RMPC é apresentada. Alguns resultados sobre RDE são revisitados na Seção 3. As soluções do FH-RMPC com garantia de estabilidade são propostas nas Seções 4 e 5. Nesta última, são utilizadas as matrizes de folga para a redução do conservadorismo da solução. Finalmente, na Seção 6, exemplos numéricos são estudados.

\section{FORMULAÇÃO DO PROBLEMA}

Considera-se o seguinte sistema linear em tempo discreto com incertezas variantes no tempo (LPV):

$\left\{\begin{array}{l}x(k+1)=\mathrm{A}(\lambda(k)) x(k)+\mathrm{B}(\lambda(k)) u(k) \\ y(k+1)=\mathrm{C} x(k+1),\end{array}\right.$

e $x(k) \in \mathbb{R}^{\mathrm{n}}$ é o estado da planta, $u(k) \in \mathbb{R}^{\mathrm{n}_{\mathrm{u}}}$ é o sinal de controle, $\mathrm{y}(k) \in \mathbb{R}^{\mathrm{n}} \mathrm{y}$ é o sinal de saída e $\lambda(k) \in \mathrm{R}^{\mathrm{L}}$ é um parâmetro incerto e variante no tempo. Supõe-se que a variável de estado $x(k)$ é mensurável no tempo $k(x(k)=$ $x(k \mid k))$. Considera-se ainda que as matrizes variantes no tempo $A(\lambda(k))$ e $B(\lambda(k))$ pertençam ao envelope convexo

$\Omega=\operatorname{Co}\left\{\left[\mathrm{A}_{1}, \mathrm{~B}_{1}\right], \ldots,\left[\mathrm{A}_{\mathrm{L}}, \mathrm{B}_{\mathrm{L}}\right]\right\}$,

com

$[\mathrm{A}(\lambda(k)) \mid \mathrm{B}(\lambda(k))]=\sum_{i=1}^{\mathrm{L}} \lambda_{i}(k)\left[\mathrm{A}_{i} \mid \mathrm{B}_{i}\right], \quad$ onde $\lambda_{i}(k) \geq$ $0, i=1, \ldots ., \mathrm{L}$ e $\sum_{i=1}^{\mathrm{L}} \lambda_{i}(k)=1$.
Para um estado inicial $x(k)$ dado, deseja-se projetar uma lei de controle MPC por realimentação de estado que garanta a estabilidade em malha fechada e minimiza $\gamma$, a cada instante de amostragem $k$ :

$$
\begin{aligned}
& \mathrm{J}_{N}(k)=\max _{[\mathrm{A}(k+j) \mid \mathrm{B}(k+j)] \in \Omega, j \geq 0} \sum_{\mathrm{j}=0}^{N-1}\left[\|x(k+j \mid k)\|_{\mathrm{Q}_{i}}^{2}+\right. \\
& \left.+\|u(k+j \mid k)\|_{\mathrm{R}_{i}}^{2}\right]+\|x(k+j \mid k)\|_{\mathrm{P}_{N}}^{2} \leq \gamma,
\end{aligned}
$$

onde $Q_{j}>0$ e $\mathrm{R}_{j}>0, \forall j \in[0, N-1]$, são matrizes de ponderação e $N$ é o horizonte de predição, satisfazendo as seguintes restrições:

$$
\begin{aligned}
& \|u(k+j \mid k)\|_{2} \leq u_{\text {máx }}, \quad \forall k \geq 0, \forall j \geq 0, \\
& \|y(k+j \mid k)\|_{2} \leq y_{\text {máx }}, \quad \forall k \geq 0, \forall j \geq 1,
\end{aligned}
$$

Este problema "min-máx" corresponde ao MPC com horizonte finito obtido com o modelo de predição que resulta no maior valor de $J_{N}(k)$ entre todos os modelos em $\Omega$.

\section{EQUAÇÕES A DIFERENÇAS DE RICCATI (RDE)}

Na solução do problema proposto (4), alguns resultados sobre a monotonicidade e estabilidade de controladores LQ (Linear Quadrático) com horizonte deslizante são utilizados (Bitmead et al. (1990), de Souza et al. (1986), de Souza (1989), Wimmer (1992)).

A solução do problema de Controle LQ com horizonte finito, para sistemas variantes no tempo, com função custo definida em (4), é dada pela sequência ótima de controle:

$u(j)=K_{j} x(j), j \in[0, N)$,

com matriz de ganho de realimentação de estados:

$K_{j}=-\left[\mathrm{R}_{j}+B_{j}^{T} \mathrm{P}_{j+1} B_{j}\right]^{-1} B_{j}^{T} \mathrm{P}_{j+1} A_{j}$,

e $P_{j}$ são as matrizes simétricas definidas positivas $\left(P_{j}>0\right)$, obtidas recursivamente pela RDE (Riccati Backward Difference Equation):

$\mathrm{P}_{j}=\mathrm{A}_{j}^{\mathrm{T}} \mathrm{P}_{j+1} A_{j}-\mathrm{A}_{j}^{\mathrm{T}} \mathrm{P}_{j+1} B_{j}\left[R_{j}+B_{j}^{T} \mathrm{P}_{j+1} B_{j}\right]^{-1} B_{j}^{T} \mathrm{P}_{j+1} A_{j}+$ $\mathrm{Q}_{j}, j \in[0, N)$,

com $P_{N}$ conhecida. O valor ótimo da função custo (4) resultante é $J_{N}(x(0))=\|x(0)\|_{P_{0}}^{2}$. Ressalta-se que no Controle LQ com horizonte deslizante apenas o sinal $u(0)$ é aplicado ao sistema em cada janela de tempo.

Os teoremas a seguir foram apresentados em Bitmead et al. (1990) e Bitmead et al. (1985), respectivamente, e adaptados para a sequência $\left\{P_{j}\right\}_{j=0}^{N}$ tratada neste artigo. 


\section{Teorema 01: (Bitmead et al.(1985))}

Se a solução $P_{j}$ definida não-negativa da RDE (9) é monotonicamente não crescente em uma determinada iteração, isto é:

$\mathrm{P}_{j+1} \geq \mathrm{P}_{j}, \quad$ para algum $j$,

então $\mathrm{P}_{j}$ é monotonicamente não crescente para todos as iterações subsequentes:

$\mathrm{P}_{j-i+1} \geq \mathrm{P}_{j-i}, \forall i \geq 0$.

O teorema a seguir (Bitmead et al. (1985)) relaciona a propriedade da monotonicidade da solução da RDE com a estabilidade assintótica de malha fechada.

Teorma 02: (Bitmead et al. (1990))

Seja a RDE em (9). Se

- $\left[\mathrm{A}_{j}, \mathrm{~B}_{j}\right]$ é estabilizável;

- $\left[\mathrm{A}_{j}, Q_{j}^{1 / 2}\right]$ é detectável;

- $\mathrm{P}_{j+1} \geq \mathrm{P}_{j}, \quad$ para algum $j$;

Então

$\overline{\mathrm{A}}_{i}=A_{i}+B_{i} K_{i}=\mathrm{A}_{i}-B_{i}\left(B_{i}^{T} P_{i+1} B_{i}+R_{i}\right)^{-1} B_{i}^{T} P_{i+1} A_{i}$ estável para todo $i \leq j$.

De fato, a partir das equações (8) e (9), tem-se:

$\left(A_{j}+B_{j} K_{j}\right)^{T} P_{j+1}\left(A_{j}+B_{j} K_{j}\right)+K_{j}^{T} R_{j} K_{j}+Q_{j}=\mathrm{P}_{j}$.

Como $\mathrm{P}_{j+1} \geq \mathrm{P}_{j}$, para algum $\mathrm{j}$, então:

$\left(A_{j}+B_{j} K_{j}\right)^{T} P_{j+1}\left(A_{j}+B_{j} K_{j}\right)+K_{j}^{T} R_{j} K_{j}+Q_{j} \leq \mathrm{P}_{j+1}$.

O que implica em:

$\left(A_{j}+B_{j} K_{j}\right)^{T} P_{j+1}\left(A_{j}+B_{j} K_{j}\right)-\mathrm{P}_{j+1} \preceq 0$.

Pelo teorema 01 e desigualdade (14), para todo $i \leq j$, o sistema em malha fechada $A_{i}+B_{i} K_{i}$ é assintoticamente estável.

\section{FH-RMPC ESTÁVEL}

Nesta seção, uma solução estável para o problema de síntese de controladores MPC robustos com horizonte finito é apresentada. A cada instante de amostragem $k$, determina-se uma lei de controle por realimentação de estados $u(k+j \mid k)=K_{j} x(k+j \mid k), \forall j \in[0, N)$, que minimiza um limitante superior da função custo (4), sujeita a restrições nos sinais de controle e saída.

Como é usual em estratégias MPC, somente o primeiro sinal de controle $u(k)=u(k \mid k)=K_{0} x(k \mid k)$ é aplicado ao sistema, e o procedimento é repetido no instante de amostragem $k+1$.
O terorema a seguir é baseado nas equações a diferenças de Riccati e suas propriedades, adotando-se uma abordagem via LMI, apropriada para uma síntese robusta.

\section{Teorema 03:}

Sejam o sistema (1) com o conjunto de incertezas associada $\Omega$ e $x(k)=x(k \mid k)$ o estado medido a cada instante de amostragem $k$. O problema FH-RMPC com garantia de estabilidade quadrática e restrições nos sinais de controle e saída pode ser resolvido pelo seguinte problema de otimização convexa, em cada instante de amostragem $k$ :

$\min \gamma$

$\gamma>0, W_{j}>0, Y_{j}$

sujeito a:

$\left[\begin{array}{ccrc}W_{j} & W_{j} A_{i}{ }^{T}+Y_{j}{ }^{T} B_{i}{ }^{T} & W_{j} Q_{j}^{\frac{1}{2}} & Y_{j}^{T} R_{j}^{\frac{1}{2}} \\ A_{i} W_{j}+B_{i} Y_{j} & W_{j+1} & 0 & 0 \\ Q_{j}^{\frac{1}{2}} W_{j} & 0 & \gamma I & 0 \\ R_{j}^{\frac{1}{2}} Y_{j} & 0 & 0 & \gamma I\end{array}\right] \geq 0$,

$\left[\begin{array}{cc}1 & x(k \mid k)^{T} \\ x(k \mid k) & W_{0}\end{array}\right] \geq 0$,

$W_{N-1} \geq W_{N}$,

$\left[\begin{array}{cc}u_{\text {máx }}^{2} & Y_{j} \\ Y_{j}^{T} & W_{j}\end{array}\right] \geq 0$,

$\left[\begin{array}{cc}y_{\text {máx }}^{2} I & C A_{i} W_{j}+C B_{i} Y_{j} \\ W_{j} C^{T}+Y_{j}^{T} B_{i}^{T} C^{T} & W_{j}\end{array}\right] \geq 0$,

com $i=1, \ldots ., L$, e $j=0,1, \ldots, N-1$. A sequência de ganhos de realimentação de estado estabilizantes é calculada então a partir da solução ótima de (15) através de $K_{j}=$ $\mathrm{Y}_{j} \mathrm{~W}_{j}^{-1}, j=0,1, \ldots, N-1$, e somente o primeiro sinal de controle $u(k \mid k)=K_{0} x(k \mid k)$ é aplicado ao sistema.

Prova:

Demonstra-se inicialmente que (16) se equivale a:

$\left(A_{i}+B_{i} K_{i}\right)^{T} P_{j+1}\left(A_{i}+B_{i} K_{i}\right)+K_{i}^{T} R_{i} K_{i}+Q_{j} \leq \mathrm{P}_{j}$,

$\forall i=1,2, \ldots, L$ e $j=0,1, \ldots, N-1$.

Definindo-se $W_{j}=\gamma P_{j}^{-1}$ e $W_{j+1}=\gamma P_{j+1}^{-1}$, com

$\overline{\mathrm{A}}_{i}=\left(\mathrm{A}_{i}+\mathrm{B}_{i} \mathrm{~K}_{i}\right)$, obtém-se a partir de (21), que: 
$\overline{\mathrm{A}}_{i}^{\mathrm{T}} \gamma \mathrm{W}_{j+1}^{-1} \overline{\mathrm{A}}_{i}-\gamma \mathrm{W}_{j}^{-1}+\mathrm{K}_{i}^{\mathrm{T}} R_{i} \mathrm{~K}_{i}+\mathrm{Q}_{j} \leq 0$.

Utilizando-se o complemento de Schur (22), resulta em:

$$
\begin{aligned}
& {\left[\begin{array}{cc}
\mathrm{W}_{j}^{-1}-\gamma^{-1} \mathrm{~K}_{i}^{\mathrm{T}} \mathrm{R}_{i} \mathrm{~K}_{i}-\gamma^{-1} \mathrm{Q}_{j} & \overline{\mathrm{A}}_{i}^{\mathrm{T}} \\
\overline{\mathrm{A}}_{i} & \mathrm{~W}_{j+1}
\end{array}\right] \geq 0,} \\
& \forall i=1,2, \ldots, L e j=0,1, \ldots, N-1 .
\end{aligned}
$$

Multiplicando-se (23) à direita e à esquerda por $\operatorname{diag}\left(W_{j}\right.$, I) e utilizando-se o complemento de Schur, com $K_{j} W_{j}=Y_{j}$, temse que:

$$
\left[\begin{array}{ccrc}
W_{j} & W_{j} A_{i}{ }^{T}+Y_{j}{ }^{T} B_{i}{ }^{T} & W_{j} Q_{j}^{\frac{1}{2}} & Y_{j}{ }^{T} R_{i}^{\frac{1}{2}} \\
A_{i} W_{j}+B_{i} Y_{j} & W_{j+1} & 0 & 0 \\
Q_{i}^{\frac{1}{2}} W_{j} & 0 & \gamma I & 0 \\
\frac{1}{2} R_{i} & 0 & 0 & \gamma I
\end{array}\right] \geq 0 .
$$

Logo a desigualdade (21) é equivalente a LMI (16). Pelos Teoremas 1 e 2, considerando-se a LMI (18) a condição (21) implica que os ganhos $K_{j}=Y_{j} W_{j}^{-1}, j=0,1, \ldots, N-1$, estabilizam o sistema em malha fechada.

A solução ótima do problema LQ é dada por $J_{N}^{*}=x(k \mid k)^{\mathrm{T}} \mathrm{P}_{0} x(k \mid k)$. Como $x(k \mid k)^{\mathrm{T}} \mathrm{P}_{0} x(k \mid k) \leq \gamma$, com $\mathrm{W}_{0}=\gamma P_{0}^{-1}$, e utilizando-se o complemento de Schur, minimizar (4) é equivalente a:

$$
\left.\begin{array}{cc}
\gamma & x(k \mid k)^{\mathrm{T}} \\
x(k \mid k) & \mathrm{W}_{0}
\end{array}\right] \geq 0 . \min _{\gamma, w_{0}>0} \gamma
$$

As LMIs (19) e (20) associadas às restrições nos sinais de controle e saída, respectivamente, são obtidas de forma similar àquelas de Kothare et al. (1996).

Cabe ressaltar, que a condição terminal não necessita ser imposta ao problema. A otimização pode ser feita $\operatorname{com} P_{N}$ fixa ou não.

Pode ser provado de forma relativamente simples que a solução do problema descrita pelo Teorema 03 é factível no instante de amostragem $\mathrm{k}$ então ela é factível em todos os instantes futuros $j>k$.

\section{FH-RMPC COM MATRIZ DE FOLGA}

Nesta seção, é utilizada a técnica proposta por Oliveira et al. (1999) e adaptada por Mao (2003), no contexto de sistemas variantes no tempo. Em Oliveira et al. (1999), os autores propuseram o uso de matrizes de folga $G$ possibilitando a redução do conservadorismo associado à estabilidade quadrática com a utilização de funções de Lyapunov dependentes de parâmetros.

O teorema a seguir apresenta uma solução estável para o FHRMPC com matrizes de folga.

\section{Teorema 04:}

Sejam o sistema incerto (1) e $x(k)=x(k \mid k)$ o estado do sistema medido no instante de amostragem $k$. Os ganhos de realimentação de estado com horizonte deslizante, em cada instante de amostragem $k$, que minimizam o limitante superior de $J_{N}(k)$ e satisfazem as restrições de controle e estado são dados pelas matrizes $K_{j}=Y_{j} G_{j}^{-1}, \forall j=$ $0,1, \ldots, N-1$, e $G_{j}, Y_{j}$ e $W_{j, i}$ são soluções do seguinte problema de otimização convexa:

$$
\min _{\gamma>0, W_{j, i}>0, Y_{j}, G_{j}}
$$

sujeito a:

$$
\left[\begin{array}{ccrc}
G_{j}+G_{j}{ }^{T}-W_{j, i} & G_{j} A_{i}{ }^{T}+Y_{j}{ }^{T} B_{i}{ }^{T} & G_{j} Q_{j}^{\frac{1}{2}} & Y_{j}^{T} R_{j}^{\frac{1}{2}} \\
A_{i} G_{j}+B_{i} Y_{j} & W_{j+1, i} & 0 & 0 \\
Q_{j}^{\frac{1}{2}} G_{j} & 0 & \gamma I & 0 \\
R_{j}^{\frac{1}{2}} Y_{j} & 0 & 0 & \gamma I
\end{array}\right] \geq 0,
$$

$$
\left[\begin{array}{cc}
1 & x(k \mid k)^{T} \\
x(k \mid k) & W_{0, i}
\end{array}\right] \geq 0
$$

$$
W_{N-1, i} \geq W_{N, i}
$$

$$
\left[\begin{array}{cc}
u_{m a ́ x}^{2} I & Y_{j} \\
Y_{j}^{T} & G_{j}+G_{j}^{T}-W_{j, i}
\end{array}\right] \geq 0,
$$

$$
\left[\begin{array}{cc}
y_{\text {máx }}^{2} I & C A_{i} W_{j, i}+C B_{i} Y_{j} \\
W_{j, i} A_{i}{ }^{T} C^{T}+Y_{j}^{T} B_{i}^{T} C^{T} & G_{j}+G_{j}^{T}-W_{j, i}
\end{array}\right] \geq 0
$$

com $i=1, \ldots, L$, e $j=0,1, \ldots, N-1$. O sinal de controle aplicado no instante de amostragem $k$ é $u(k \mid k)=K_{0} x(k \mid k)$.

Prova: a demonstração deste teorema pode ser feita a partir do teorema 03 e dos resultados sobre matriz de folga (Oliveira et al. (1999)), e serão omitidas devido à falta de espaço.

A factibilidade e estabilidade podem ser provadas de forma similar àquelas do teorema 03.

\section{EXEMPLO NUMÉRICO}

\subsection{Exemplo 01}

Considera-se o sistema linear variante no tempo (Ding et al.2004):

$$
\left[\begin{array}{l}
x_{1}(k+1) \\
x_{2}(k+1)
\end{array}\right]=\left[\begin{array}{cc}
1 & 0 \\
\mathrm{~K}(k) & 1
\end{array}\right]\left[\begin{array}{l}
x_{1}(k) \\
x_{2}(k)
\end{array}\right]+\left[\begin{array}{l}
1 \\
0
\end{array}\right] u(k)
$$

onde $\mathrm{K}(k)$ é um parâmetro variante no tempo. A condição inicial é $x_{0}=\left[\begin{array}{ll}2 & 2\end{array}\right]^{\mathrm{T}}$, as matrizes de ponderação são $\mathrm{Q}=\mathrm{I}$ e $\mathrm{R}=1$, a restrição no sinal de controle é $|u(k)| \leq 1, N=4$, 
e a saída é dada por $y(k)=x_{2}(k)$.

Caso 1: a matriz dinâmica do sistema apresenta incertezas paramétricas com $K \in\left[0,01, K_{M}\right]$. $\mathrm{O}$ valor do parâmetro $\mathrm{K}_{\mathrm{M}}$ é deixado livre para testar a factibilidade do método proposto.

Caso 2: considerando-se o mesmo sistema do caso 1 com $\mathrm{K}(k)=1,5+\operatorname{sen}(k)$ para calcular a evolução dos estados e $0,5 \leq K_{M} \leq 2,5$ para a formar o politopo.

Inicialmente, foi realizado um teste para determinar o maior valor de $K_{M}$ (caso 01) para o qual as técnicas IH-RMPC, de Cuzzola et al. (2002) e Ding et al. (2004), e a FH-RMPC, proposta neste trabalho, todas com matrizes de folga, apresentam soluções factíveis. Utilizando-se $\mathrm{K}=2,5$ nas simulações, foi observado que para as técnicas IH-RMPC de Cuzzola et al. (2002) e Ding et al. (2004) não há solução factível para valores de $\mathrm{K}_{\mathrm{M}}>78$ e $\mathrm{K}_{\mathrm{M}}>105$, respectivamente, enquanto que para a técnica de FH-RMPC proposta neste trabalho, a infactibilidade acontece para $\mathrm{K}_{\mathrm{M}}>124$. Estes resultados podem ser vistos na Tabela 1 .

Tabela 1. Comparação do $K_{M}$ máximo

\begin{tabular}{|c|c|c|c|}
\hline & FH-RMPC & $\begin{array}{c}\text { IH-RMPC } \\
\text { Ding } \text { et al. } \text { (2004) }\end{array}$ & $\begin{array}{c}\text { IH-RMPC } \\
\text { Cuzzola } \text { et al. (2002) }\end{array}$ \\
\hline$K_{M}$ & 124 & 105 & 77 \\
\hline
\end{tabular}

Em seguida, foram realizadas simulações para o caso 02 utilizando-se a técnica IH-RMPC de Cuzzola et al. (2002), considerando-se as observações feitas por Mao (2003) para sistemas variantes no tempo, e a técnica FH-RMPC proposta neste trabalho. Observa-se na Figura 1, que em ambos os casos os estados convergem para a origem. Na figura 2, verifica-se que é respeitada a restrição de $|u(k)| \leq 1$. Na Figura 3, observa-se os valores de $\gamma$ das duas técnicas.

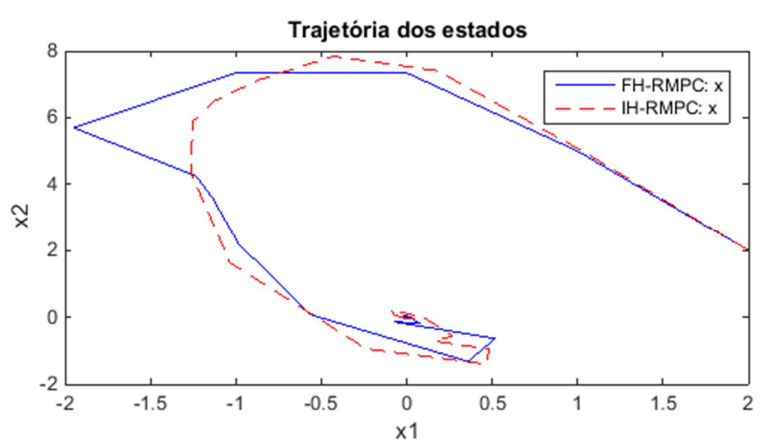

Fig. 1-Trajetória dos estados FH-RMPCxIH-RMPC(caso 02)

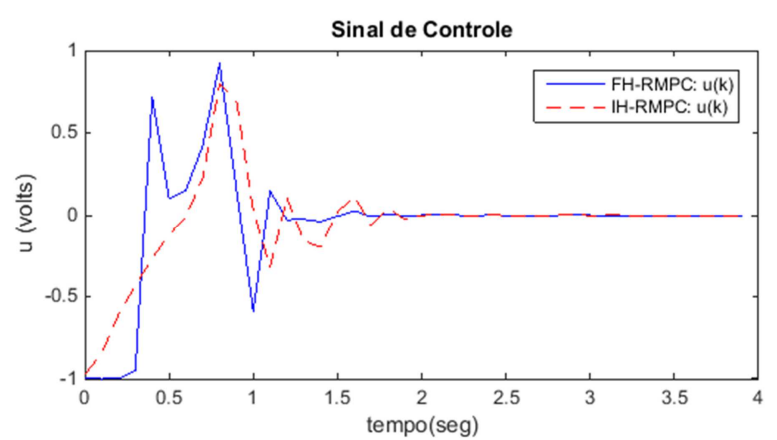

Fig. 2-Sinal de controle FH-RMPC x IH-RMPC (caso 02)

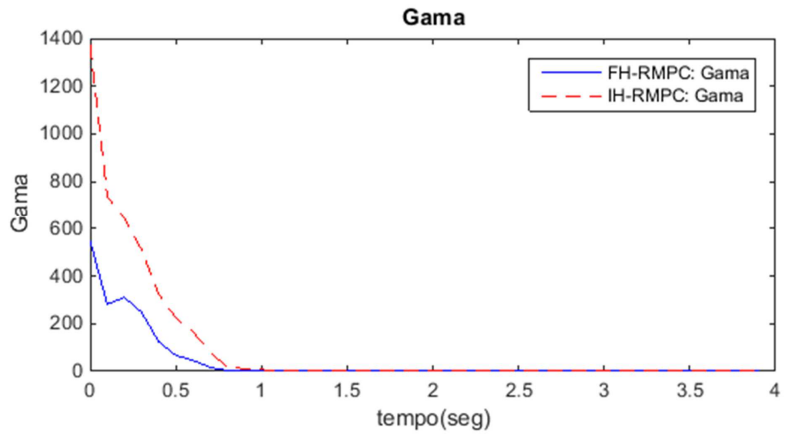

Fig. 3 - Coeficiente de performance $\gamma$ FH-RMPC x IHRMPC (caso 02)

Para a comparação das técnicas FH-RMPC e IH-RMPC de forma quantitativa foram utilizados os indíces de desempenho baseados no erro IAE, ITAE, ISE e ITSE comuns na literatura (Duarte-Mermoud e Prieto, 2004) e o índice Variação total de Controle (TCV - Total variation of Control) no intervalo de 0 a 4 segundos.

Comparando-se os valores dos índices de desempenho obtidos (ver Tabela 2), a estratégia FH-RMPC possui índices IAE, ITAE e ITSE inferiores ao da estratégia IH-RMPC, entretanto apresenta ISE, na análise do $x_{1}$, e esforço de controle, observado pelo índice TCV, mais elevados,.

Tabela 3. Índices de desempenho

\begin{tabular}{|c|c|c|c|c|c|c|}
\hline \multicolumn{2}{|c|}{ Método } & IAE & ITAE & ISE & ITSE & TCV \\
\hline \multirow{2}{*}{ FH-RMPC } & $x_{1}$ & 11,01 & 50,05 & 12,31 & 49,88 & \multirow{2}{*}{4,73} \\
\cline { 2 - 7 } & $x_{2}$ & 39,96 & 149,93 & 205,25 & 648,27 & \\
\hline \multirow{2}{*}{ IH-RMPC } & $x_{1}$ & 11,16 & 70,85 & 12,16 & 59,23 & \multirow{2}{*}{4,33} \\
\cline { 2 - 6 } & $x_{2}$ & 52,73 & 291,42 & 299,26 & 1443,1 & \\
\hline
\end{tabular}

\subsection{Exemplo 02}

O segundo estudo de caso é o sistema de controle de posição angular Kothare et al. (1996). O movimento da antena é descrito pela seguinte equação em tempo discreto:

$$
\begin{gathered}
x(k+1)=\left[\begin{array}{cc}
1 & 0,1 \\
0 & 1-0,1 \alpha(\mathrm{k})
\end{array}\right] x(k)+\left[\begin{array}{c}
0 \\
0,0787
\end{array}\right] u(k) \\
y(k)=\left[\begin{array}{ll}
1 & 0
\end{array}\right] x(k)
\end{gathered}
$$

O parâmetro $\alpha(k)$ é proporcional ao coeficiente de fricção viscosa e varia arbitrariamente no intervalo $0.1 \mathrm{sec}^{-1} \leq \propto$ $(k) \leq 10 \mathrm{sec}^{-1}$.

O estado inicial é $x(0)=\left[\begin{array}{c}0,05 \\ 0\end{array}\right]$, as matrizes de ponderações da função custo são $Q=C^{T} C$ e $\mathrm{R}=0,00002$, e a restrição de sinal de controle é dado por $|u(k+i \mid k)| \leq 2$ volts e o horizonte de predição $N=3$.

Foram realizadas simulações para o sistema LTV com $\alpha(k)$ variando uniformemente no tempo, no intervalo entre $[0,1 ; 10]$, utilizando os algoritmos FH-RMPC e a técnica IHRMPC baseado em Cuzzola et al. (2002)/Mao (2003), ambos com matrizes de folga. Foi observado a partir da Figura 4, que em ambos os casos os estados convergem para a origem. No gráfico do sinal de controle pode ser observado que é respeitada a restrição de $|u(k)| \leq 2$. 


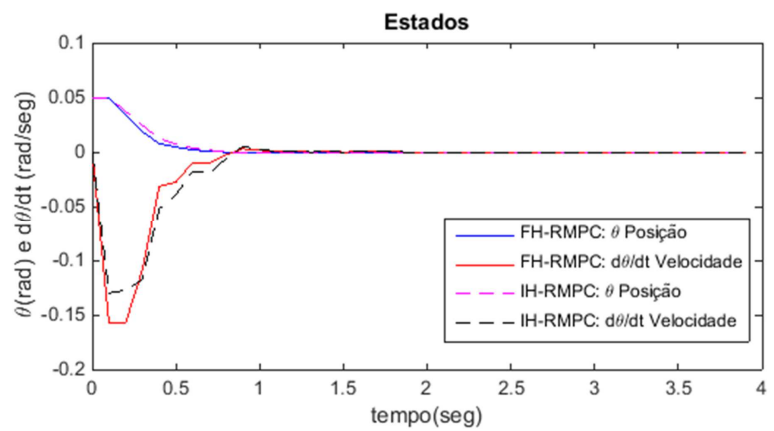

Fig. 4 -Estados FH-RMPC x IH-RMPC

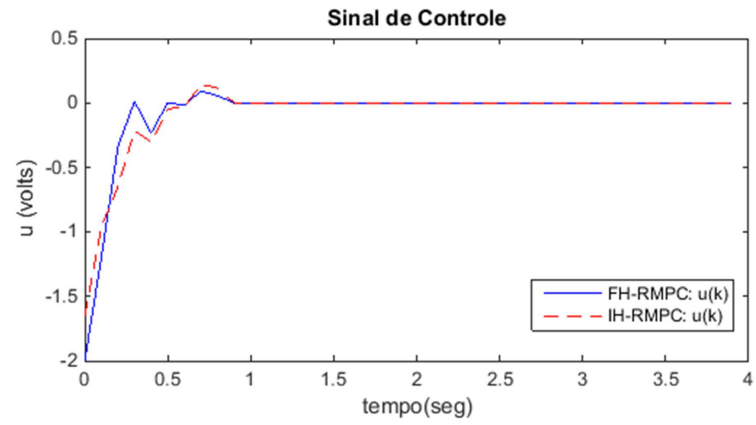

Fig. 5 -Sinal de controle FH-RMPC x IH-RMPC

Comparando-se os índices de desempenho apresentados na Tabela 4, a estratégia FH-RMPC possui valores menores para a maioria dos índices.

Tabela 4. Índices de desempenho

\begin{tabular}{|c|c|c|c|c|c|c|}
\hline \multicolumn{2}{|c|}{ Método } & IAE & ITAE & \multicolumn{1}{|c|}{ ISE } & ITSE & TCV \\
\hline \multirow{2}{*}{ FH-RMPC } & $x_{1}$ & 0,17 & 0,42 & 0,0066 & 0,013 & \multirow{2}{*}{2,74} \\
& $x_{2}$ & 0,51 & 1,77 & 0,063 & 0,18 & \\
\hline \multirow{2}{*}{ IH-RMPC } & $x_{1}$ & 0,19 & 0,52 & 0,0072 & 0,015 & \multirow{2}{*}{2,15} \\
\cline { 2 - 7 } & $x_{2}$ & 0,52 & 2,040 & 0,052 & 0,17 & \\
\hline
\end{tabular}

\section{CONCLUSÕES}

Uma nova abordagem para Controle Preditivo Robusto baseado em Modelo de Horizonte Finito para sistemas lineares com incertezas politópicas variantes no tempo foi apresentada. Devido à propriedade de monotonicidade das RDEs, a sequência de controle obtida como solução do problema de otimização estabiliza o sistema em malha fechada. Nesta abordagem, não são utilizadas restrições terminais, embora o problema ainda possa ser resolvido com uma matriz de custo terminal fixa. Exemplos numéricos de sistemas com incertezas variantes no tempo demonstraram o bom desempenho do controlador proposto, e, quando comparado com uma técnica IH-RMPC, mostrou-se mais robusto em relação às incertezas paramétricas.

\section{REFERÊNCIAS}

Bitmead, R. R.; Gevers, M.; Wertz, V. (1990); Adaptive Optimal Control: The Thinking Man's GPC. Prentice Hall. Englewood Cliffss, NJ.

Bitmead, R.; Gevers, M.; Petersen, I. R.; Kaye, R. J. (1985); Monotonicity and stabilizability Properties of Solutions of Riccati Difference Equation. Systems and Control Letters, vol. 5, pp 309-315.
Cassavola, A.; Formularo, D.; Franzé, G. (2004); Robust Constrained predictive control of uncertain normbounded linear systems. Automática, 40, pp.1865-1876.

Chu, D.; Chen, T.; Marquez, H. J. (2006); Finite horizon robust model predictive control with terminal cost constraints, IEE Proceedings D: Control Theory Applications, 153 , pp. 156-166.

Cuzola, F.; Geromel, J. C.; Morari. M.(2002); An improved apropproach for constrained robust model predictive control. Automatica , 38, pp.1183-1189.

De Souza, C. E.; Gevers, M.; Goodwin, G. C. (1986); Riccati Equations in Optimal Filtering of Nonstabilizable Systems having Singular State Transition Matrices. IEEE Transactions on Automatic Control, vol. AC-31, pp 831838.

Duarte-Mermoud, M.A. y Prieto, R.A. (2004). A Performance index for quality response of dynamical systems. ISA Transactions 43, 133-151.

De Souza, C. E (1989); Monotonicity and Stabilizability results for the solution of the Riccati Difference Equation. Proc. Workshop on the Riccati Equation in Control, Systems and Signals, S Bittanti (ed.), Como, Italy, pp. 38-41.

Ding, B.; Xi, Y.; Li, S. (2004); A Synthesis approach of online constrained robust model predictive control. Automática, 40, pp.163-167.

Kothare, M. V.;Balakrishman, V.; Morari. M. (1996); Robust Constrained Model Predictive Control using Linear Matrix Inequalities, Automatica, 32, pp.1361-1379.

Mao, W. (2003); Robust stabilization of uncertain timevarying discrete systems and comments on "an improved approach for constrained robust model predictive control”. Automatica, 39, pp.1109-1112.

Oliveira, M. C.; Bernussou, J.; Geromel, J. C. (1999); A new discrete-time robust stability condition. Systems \& Control Letters, 37, pp.261-265.

Kim, K. B.; (2002); Implementation of stabilizing receding horizon controls for time-varyng systems. Automática, 38, pp.1705-1711.

Wada, N.; Saito, K.; Saeki, M. (2006); Model Predictive Control for Linear Parameter Varyng Systems Using Parameter Dependent Lyapunov Function. IEEE Transactions on Circuits and Systems, 53, $\mathrm{n}^{\circ} 12$.

Wimmer, H. K. (1992); A Comparison theorem for matrix Riccati difference equations. Systems \& Control Letters, North-Holland, 19, pp.223-239. 